\title{
Laterality effects and choice reaction time in a unimanual two-finger task ${ }^{1}$
}

\author{
J. L. BRADSHAW ${ }^{2}$ AND A. D. PERRIMENT \\ MONASH UNIVERSITY
}

\begin{abstract}
A unimanual, two-digit choice-reaction-time task was used in conjunction with visual signals presented in the extreme peripheral field. The position of the signal on the retina, the eye stimulated, the hand used in responding, and the operating digits were all controlled for their effects. Stimulus-response compatibility was maintained throughout. It was found that RT to ipsilateral $S-R$ pathways was consistently faster than when the pathways involved transcommissural connections. Subsidiary findings related to differences between the operating digits and the side of signal occurrence. These effects are discussed in connection with known interhemispheric phenomena and other $C R T$ findings.
\end{abstract}

In a typical choice-reaction-time (CRT) task, the $S$ monitors two horizontally displaced signals and responds by pressing corresponding buttons. The best strategy for the $S$ to adopt, particularly when the signals are widely displaced and occur with equal probability, is to fixate a midpoint between thern. In such situations, there is a greater likelihood of the signal falling on one hemiretina rather than on the other and always arriving first by a direct route at one cerebral hemisphere. It is suggested that the transcommissural pathways known to link the separate cerebral hemispheres could affect performance significantly. As different sensory pathways and muscle groups may be involved in the two responses, there is the additional possibility of confounding through neuromuscular differences and hemiretinal and interocular effects.

Figure 1 shows why it is natural and compatible to respond to a left signal with the left hand and to a right signal with the right. There is abundant anatomical evidence (Myers, 1956, 1962, 1965; Sperry, 1962, 1964) that the activity of each hand, and its somesthetic and visual control, are highly lateralized in the opposite hemisphere. The brain can be viewed as two mirror systems, each tuned to one lateral half of the space confronting the S. Figure 1 also shows that, irrespective of whether one or both eyes are used, a signal to the left of fixation travels via the right hemiretina directly to the right cerebral cortex, and a signal on the right travels first to the left hemisphere. This follows from the arrangement of fibers

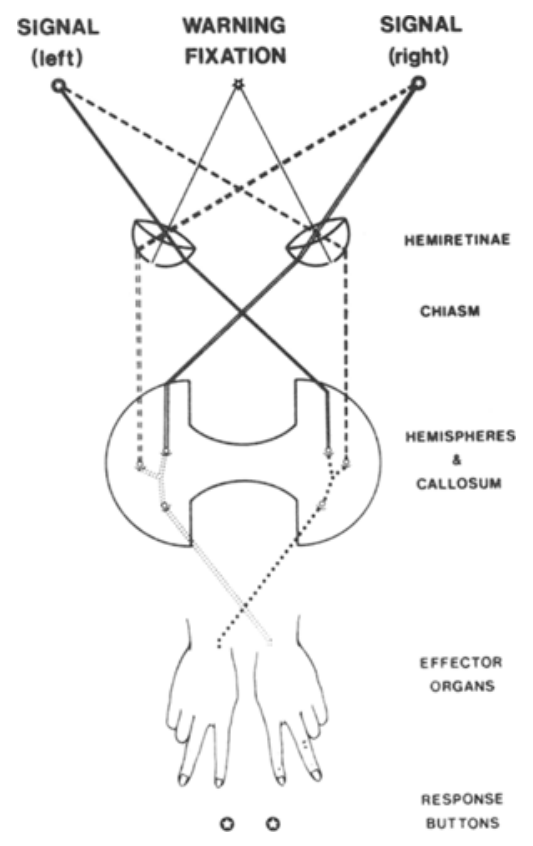

Fig. 1. Schematic representation of the physiological system, showing ipsilateral pathways. The contralateral sensory-motor roate across the corpus callosum (not shown) is taken if a left hand is responding when a right signal occurs, or vice versa.

from the temporal hemiretinae traveling directly to the ipsilateral hemispheres and those from the nasal hemiretinae crossing via the chiasma to the contralateral projection areas.

Jeeves (1965) examined the RT of several congenitally acallosal Ss, i.e., born without the major intercortical commissure, together with a number of normals for control purposes. Ss responded to either of two pea lights, which were mounted on spectacle frames, by depressing a button held in one hand. He found considerable differences in RT between groups and ascribed this result to the involvement of either ipsilateral or contralateral pathways. This finding invites closer examination. It has been shown that RT is dependent upon the retinal locus of an incident signal (Poffenberger, 1912; Rains, 1963; Payne, 1966). There is also more recent evidence ${ }^{3}$ that in a unimanual task, where two digits respond in a CRT paradigm, the forefinger (Digit 2) responds faster than the middle finger (Digit 3). The question arises as to whether or not these findings are independent of transcommissural effects. Accordingly, an experiment was designed to examine systematically the effect upon RT of such retinal and cortical factors.

A unimanual CRT task was chosen in which the second and third digits of each hand responded to signals that were presented peripherally to either eye. The main effects controlled were the position of the signal on the retina, the eye stimulated, the hand used for responding, and the operating digit. Although the signals and response buttons were laterally paired, the relationship of the operating digits was reversed by having the operating hand held either in pronation or in supination. This was facilitated by suspending the response panel so that the buttons could be operated either from above or below. Consequently, the four digits could be response-linked with the two signals in all combinations of direct and indirect sensory-motor pathways without interfering with the $S-R$ expectancies of the $S$.

\section{Apparatus \\ METHOD}

Two stimulus lamps, 34 in. apart, were located one on each side of the $S$ at an angle of $90 \mathrm{deg}$ from the median plane. A third lamp was set 17 in. directly in front and acted both as a ready signal and as a fixation point. Each of the lamps was set behind a metal hood in the center of which a 1/32-in-diam hole provided a point source of light. A red filter covering the fixation lamp and grey filters covering the stimulus lamps prevented filament glare and insured a diffused light source. The luminance of the stimulus lamps was $31 \mathrm{ft}-\mathrm{L}$, as measured at the position of the S's eye with a SAE photometer. The peripheral position of the stimulus lamps was such that each could be viewed only monocularly, with the light projecting onto the corresponding nasal retina. The entire visual display was set into a cabin $3 \mathrm{ft}$ square and $3 \mathrm{ft}$ deep, the front of which was open. All interior surfaces were painted matte black. An adjustable chinrest, midway between the two stimulus lamps, standardized the position of the S's head. The pushbuttons with which the S responded were arranged on the back of a $3 \times 2$ in. panel. This was suspended 11 in. 

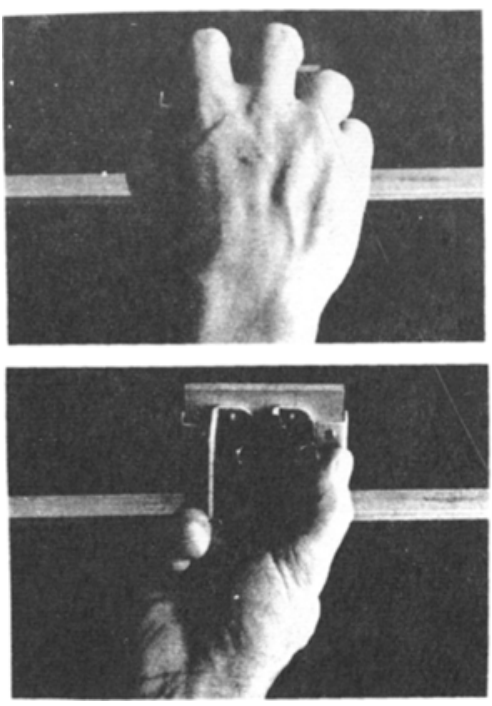

above the surface of the table upon which the stimulus cabin rested. It was inclined $5 \mathrm{deg}$ from the vertical and positioned 13 in. in front of the S's chinrest. It could be operated with equal ease either from above or below. The pushbuttons had concave surfaces and were adjusted so that they could be operated by a depression of .015 in. Figure 2 illustrates the operating position of the hands under the two response conditions.

The presentation of signals and the recording of responses were controlled by a PDP-8/S computer located in a room adjacent to the S's station. RTs to individual signals were calculated on-line and recorded progressively on the computer Teletype to the nearest $1 / 10 \mathrm{msec}$. This procedure enabled a continuous monitoring of the S's performance. A closed-circuit TV system, with the receiver at the computer console, permitted visual monitoring of the S's position and the manner in which he was operating the response buttons. A two-way communication system linked the $S$ and $E$. Ss wore a headset with noise-attenuating earmuffs, and white noise $(68 \mathrm{~dB})$ was relayed into the $S$ 's room to provide a standard auditory background.

\section{Subjects}

There were 12 male and female Ss. Some were paid undergraduate volunteers and others were members of the academic staff. None had previous experience of the task, and all were naive concerning the aims of the experiment.

\section{Procedure}

Each $S$ performed under four conditions of responding, which were counterbalanced for practice effects, using a 4 by 4 Latin square. The allocation of Ss triplicated
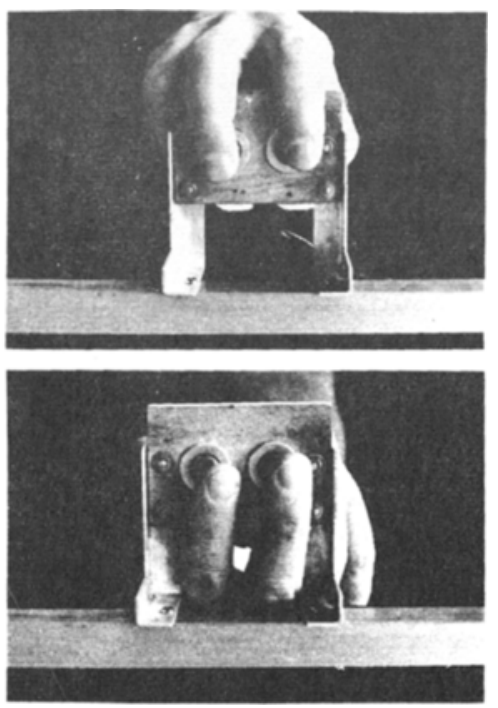

Table 1

Inter Hemispheric Pathways for the Experimental Conditions

\begin{tabular}{ccccc}
\multicolumn{5}{c}{ Experimental Conditions } \\
\hline & \multicolumn{4}{c}{ Condition } \\
\cline { 2 - 5 } Digit & L(T) & L(B) & R(T) & R(B) \\
\hline 2 & C & I & C & I \\
3 & I & C & I & C \\
\hline
\end{tabular}

$C=$ contralateral pathway $R=$ right hand $I=$ ipsilateral pathway $\quad(T)=$ in pronation $L=$ left hand

$(B)=$ in supination

each row of the square. In all conditions, the second and third digits were used to operate the response buttons. The four response conditions were as follows:

(1) $L(T)$. The left hand was heid in pronation with the responding digits operating the pushbuttons from the top of the panel. In this condition, the second digit responded to signals on the right and the third digit to signals on the left.

(2) $L(B)$. The left hand was held in supination and operated the response buttons from below the panel. This represented a reversal of the S-R pattern of Condition 1.

(3) R(T). The right hand operated the buttons from above.

(4) R(B). The right hand operated the buttons from below.

These conditions exhaust the permutations of sensory-motor pathways found in the two cortical hemispheres for this task. These relationships are summarized in Table i.

Each experimental session consisted of five blocks of 50 signals each. Left and right signais were given an overall random but equiprobable presentation. The first block was used as a training session, Ss being given 12 signals under each of the four response conditions. The order in
Fig. 2. Position of the hand on the response panel in supination and in pronation, viewed from in front and from behind.

which they were given the training trials was the same as that in which they took the four experimental conditions. A rest pause of $2 \mathrm{~min}$ was given between blocks. The sequence of signal presentation was as follows. The red warning lamp flashed for $500 \mathrm{msec}$ as a ready signal and fixation point. Two seconds later, one or the other of the peripheral signal lamps was illuminated and remained on until the $S$ made the correct response. After a 2-sec delay, which was occupied by the computer printout of the RT, the sequence was repeated until the end of the block. Following the response to the last signal in the block, three clearly audible pips were presented through the S's earphones indicating the commencement of the rest pause. Two minutes later, a further three pips alerted him for the start of the next block. When the $S$ was comfortably positioned for the next response condition, the computer was restarted and the procedure continued. At the end of the final block, a continuous tone of 5 -sec duration signaled the end of the experiment. Prior to the commencement of each session, Ss were read a standard set of instructions, and the four response conditions were demonstrated. They were also given a printed list of the order in which they were to take the conditions. The instructions emphasized the requirement for responding as rapidly as possible and also for the maintenance of a high level of concentration throughout the test blocks. They were also instructed to rest their eyes by closing them during the rest pauses.

\section{RESULTS}

Figure 3 gives the mean RTs for the ipsilateral and contralateral conditions for each of the 12 Ss. With only one exception, RT for the contralateral condition was slower than it was for the ipsilateral condition. Figure 4(a) graphs the overall means separately for the two digits and two conditions of cortical laterality. Differences in RT for ipsilateral and contralateral conditions were examined for Digits 2 and 3 separately, using the t test for correlated means. For both digits separately, ipsilateral conditions produced faster RTs $(p<.005$, one-tailed test). When the means of the two digits were combined, $p<.0005$ was obtained. Tests were also made on two other sets of data. These were RT to left and right signals and for the two digits, irrespective of different 


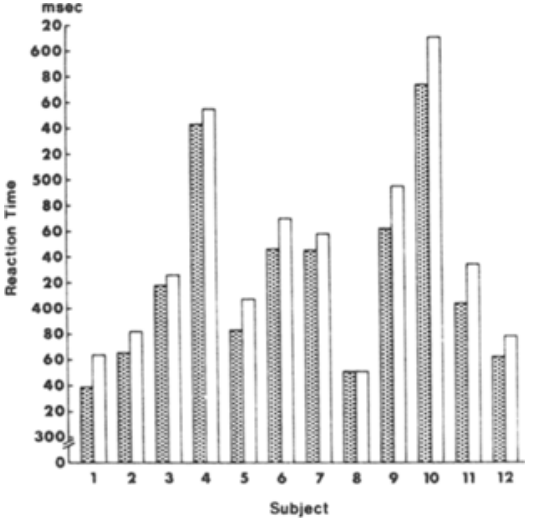

Fig. 3. Histogram of response times for the $12 \mathrm{Ss}$, showing ipsilateral (shaded) and contralateral means.

cortical pathways. In both cases, significant differences were obtained from the $t$ tests ( $p<.05$, two-tailed test). Performance differences were not obtained between left and right hands or between use in pronation and supination.

The means for the various analyses performed are plotted in Fig. 4. It can be seen that RT using Digit 2 was faster than that for Digit 3, and that left signals were associated with faster RTs than were signals on the right. It is also evident that the greatest difference in performance was associated with the two conditions of cortical laterality. The contralateral-pathway condition produced a mean RT $20 \mathrm{msec}$ greater than the ipsilateral, which represents an increase of approximately $5 \%$.

\section{DISCUSSION}

One interesting feature of the results of this experiment is that RT, where a contralateral sensory-motor pathway was
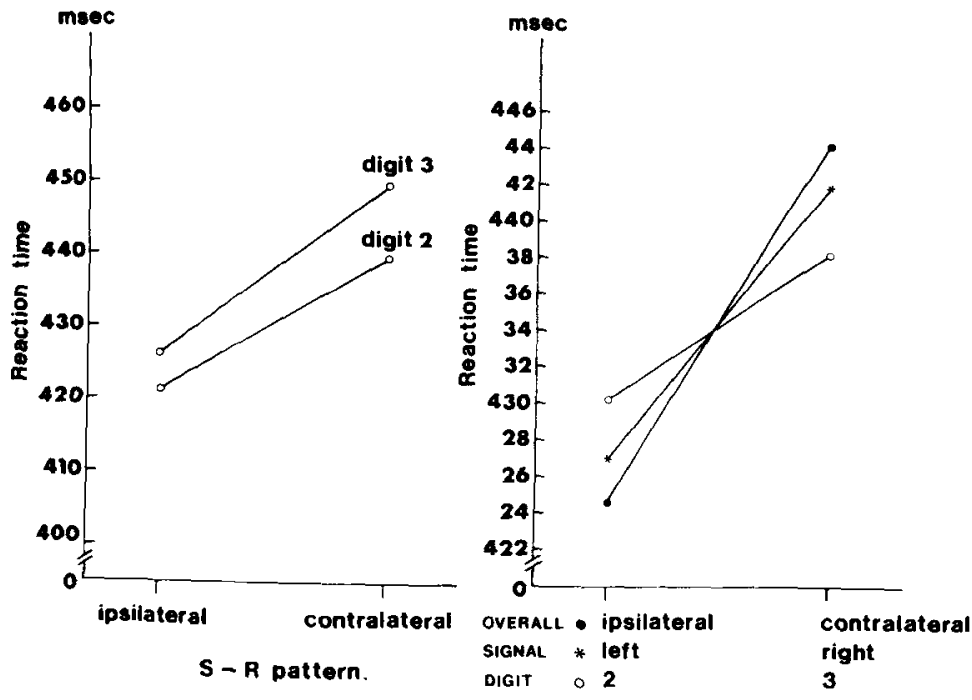

involved, was significantly slower than where there was no transcommissural crossing. The difference between the two RTs was much smaller, however, than that previously reported by Jeeves for normal Ss (Jeeves, 1965). From the data he provides, a difference of $113 \mathrm{msec}$ can be obtained. His reported difference of $113 \mathrm{msec}$ represents an increase of approximately $29 \%$. He obtained an ipsilateral mean fractionally smaller and a contralateral mean considerably larger than those obtained in the present study. The tasks used in the two studies differed, however, in important respects. It is of interest to note that Rutledge and Kennedy (1960), using direct electrical stimulation of one cortex in cats and recording from the other, obtained a transcallosal transmission delay of $40.65 \mathrm{msec}$, which approaches more closely that reported here. It must be emphasized, however, that in RT studies comparing the effects of ipsilateral and contralateral pathways, the discrepancies obtained may reflect much more than the transmission of a signal across the corpus callosum. There are other commissural pathways and possibly subcortical routes (Glickstein, 1965), particularly for such simple events as brightness discrimination. With complex, symbolic material, leaving aside the question of laterality and cortical dominance, such discrepancies might well be increased.

In relation to the present study, it could be argued that a response from the left of two digits on the right hand to a left signal or a response from a right digit on the left hand to a right signal is psychologically less compatible than when digit, limb, and signal are all laterally consistent. When questioned after the experiment, Ss did not support such an argument.

Two other findings deserve comment.
The first is consistent with results previously obtained from a different experimental task (Perriment ${ }^{3}$ ). Digit 2 again gave faster RTs than did Digit 3, although in the present study all possible sensory-motor variables were controlled. This effect can reasonably be assumed to have neuromuscular origins. In the second place, a left signal projecting to the right hemisphere gave significantly faster RTs than did a right signal. This also replicates Perriment's finding, although the S-R arrangement in the two studies was markedly dissimilar.

Teuber (1962) and Luria (1966) review the part played by the two cerebral hemispheres in sensory-motor and cognitive performance. The dominance of the left hemisphere in integrating symbolic information has long been known (Zangwill, 1960). The possibility remains that the right, or nondominant, hemisphere differs in its ability to handle simple sensory-motor response patterns (Hécaen, 1962). Whether a faster RT is obtainable from symbolic material projected to the left hemisphere is not known. There is evidence that this might be the case from work on tachistoscopic recognition of letters (Markowitz \& Weitzman, 1969; Overton \& Wiener, 1966).

\section{REFERENCES}

GLICKSTEIN, M. Interhemispheric transfer, macular sparing and the central visual pathways. In E. G. Ettlinger (Ed.), Functions of the corpus callosum. London: Churchill, 1965. Pp. 18-23.

HÉCAEN, H. Clinical symptomatology in right and left hemispheric lesions. In $V$. $B$. Mountcastle (Ed.), Interhemispheric relations and cerebral dominance. Baltimore: Johns Hopkins Press, 1962. Pp. 215-243.

JEEVES, M. A. Psychological studies of three cases of congenital agenesis of the corpus callosum. In E. G. Ettlinger (Ed.), Functions of the corpus callosum. London: Churchill, 1965. Pp. 73-94.

LURIA, A. L. Higher cortical functions in man. New York: Basic Books, 1966.

MARKOWITZ, H., \& WEITZMAN, D. $O$. Monocular recognition of letters and Landolt $\mathrm{Cs}$ in left and right visual hemifields. Journal of Experimental Psychology, 1969, 79, 187-189.

MYERS, R. E. Function of the corpus callosum in interocular tranefer. Brain, 1956, 79, 358-363.

MYERS, R. E Transmission of visual information within and between the hemispheres: A behavioral study. In V. B. Mountcastle (Ed.), Interhemispheric relations and cerebral dominance. Baltimore: Johns Hopkins Press, 1962. Pp. 51-73.

Fig. 4. (a) Mean response times, ipsilateral and contralateral, for the two digits. (b) Combined mean response times comparing the two overall cortical pathways, sides of signal occurrence and digits. 
MYERS, R. E. The neocortical commissures and interhemispheric transmission of information. In E. G. Ettlinger (Ed.), Functions of the corpus callosum. London: Churchill, 1965. Pp. 1-17.

OVERTON, W., \& WENER, M. Visual field position and word-recognition threshold. Journal of Experimental Psychology, 1966, 71, 249-253.

PAYNE, W. H. Reaction time as a function of retinal locus. Vision Research, 1966, 6, 729-732.

POFFENBERGER, A. T. Reaction time to retinal stimulation with special reference to time lost in conduction through nerve centers. Archives of Psychology, 1912, 3, 1-73.

RAINS, J. D. Signal luminance and position effects in human reaction time. Vision Research, 1963, 3, 239-251.
RUTLEDGE, L. T., \& KENNEDY, T. T. Extracallosal delayed responses to cortical stimulation in chloralosed cats. Journal of Neurophysiology, 1960, 23, 188-195.

SPERRY, $R$. W. Some general aspects of interhemispheric interpretation. In V. B. Mountcastle (Ed.), Interhemispheric relations and cerebral dominance. Baltimore: Johns Hopkins Press, 1962. Pp. 43-49.

SPERRY R. W. The great cerebral commissure. Scientific American, 1964, 210, 42-52.

TEUBER, H. K. Effects of brain wounds implicating right or left hemisphere in man: Hemisphere differences and hemisphere interaction in vision, audition and somesthesis. In V. B. Mountcastle (Ed.), Interhemispheric relations and cerebral dominance. Baltimore: Johns Hopkins Press, 1962. PF. 131-157.
ZANGWILL, O. L. Cerebral dominance and its relation to psychological function. Edinburgh: Oliver \& Boyd, 1960.

\section{NOTES}

1. This work was supported by ARGC Grant 67/16377 held by the second author.

2. Address: Monash University, Victoria, Australia.

3. Perriment, A. D. The effect of signal elements and response mode on a visual CRT task. In preparation.

(Accepted for publication June 9, 1969.) 